\title{
ON FITTING CLASSES THAT ARE SCHUNCK CLASSES
}

\author{
JOHN COSSEY
}

(Received 25 June 1980)

Communicated by H. Lausch

\begin{abstract}
An example is given to show that a class of finite soluble groups that is both a Fitting class and a Schunck class need not be a formation. The novel feature of this class is that it is defined by imposing conditions on complemented chief factors of groups in it: this technique usually does not give rise to Fitting classes that are not formations.
\end{abstract}

1980 Mathematics subject classification (Amer. Math. Soc.): 20 D 10.

The behaviour of Fitting classes of finite soluble groups under additional closure properties has been extensively studied in recent years. Those Fitting classes that are also Schunck classes seem to have escaped attention so far, and our aim here is to give an example to show that such a class need not be a formation. The example is given, as Schunck classes often are, by imposing restrictions on the complemented chief factors of groups in the class. (All groups considered in this paper will be finite and soluble.) It is the fact that the prescription leads to a Fitting class which is not a formation that makes this class novel: the prescription is just another variation on an idea of Hawkes (1970). That a class of metanilpotent groups which is both a Fitting class and a Schunck class is a formation is a consequence of Theorem 4.1 of Bryce and Cossey (1974); the groups of nilpotent length three in the example below also form a Fitting class that is a Schunck class but not a formation.

Since much of the detail below is similar to that of section 2 of Hawkes (1970), we assume familiarity with that paper, and adopt the notations and conventions therein. Moreover, we suppress details that do not differ significantly from those of Hawkes (1970).

(OCopyright Australian Mathematical Society 1981 
The class is defined in the following way. Let $p$ be a fixed prime, $Z_{p}$ the field of $p$ elements, $k$ the algebraic closure of $\mathbf{Z}_{p}$. For a group $G$, and a $\mathbf{Z}_{p}[G]$-module $U$, we have that $k \otimes_{\mathbf{z}_{p}} U$ is the direct sum of irreducible $k[G]$-modules, all of the same dimension (Curtis and Reiner (1962) Theorem 70.15): we call this dimension the absolute degree of $U$. We then define $c_{p}(G)$ to be the least common multiple of the absolute degrees of the complemented $p$ chief factors of $G$. The classes of all $p$-groups and all $p^{\prime}$-groups are denoted by $S_{p}$ and $S_{p^{\prime}}$ respectively, and $X=\mathbf{S}_{p} \mathbf{S}_{p}, \mathbf{S}_{p}$ is the usual class product. We set

$$
\mathbf{Y}=\left\{G \in \mathbf{X}: c_{p}(G) \text { is coprime to } p\right\}
$$

That $Y$ is a Schunck class is clear from its definition; we show that it is a Fitting class, but not a formation.

To see that $\mathrm{Y}$ is $N_{0}$-closed, it is enough, by a standard argument, to prove that if $G=N_{1} N_{2}$, with each $N_{i}$ a maximal normal subgroup of $G$ and in $\mathrm{Y}$, then $G$ is in $Y$. Since $G$ is then clearly in $X$, it is enough to show that each complemented $p$ chief factor has $p^{\prime}$ absolute degree. By Theorem 1 of Barnes (1972), it will be enough to check this on the complemented chief factors in a fixed chief series: we choose a chief series $G \triangleleft N_{1} \triangleleft N_{1} \cap N_{2}=M_{1} \triangleleft \cdots \triangleleft M_{n}=1$. Since $G / N_{1}$ and $N_{1} / N_{1} \cap N_{2}$ are cyclic, we need only consider complemented chief factors $M_{i} / M_{i+1}$ with $M_{i}<N_{1} \cap N_{2}$. Then, noting that if $M_{i} / M_{i+1}$ is a complemented chief factor of $G$, it is a direct product of complemented chief factors of $N_{j}, j=1,2$, the arguments of Hawkes (1970) apply to complete the proof.

For $S_{n}$-closure, it is enough to show that if $G$ is in $Y$, and $N$ is a maximal normal subgroup of $G$, then $N$ is in $\mathrm{Y}$. Again, $N$ is in $\mathrm{X}$, and so it will be enough to show that the complemented $p$-chief factors of $N$ have $p^{\prime}$ absolute degree. By the definition of $\mathbf{X}$, it will be enough to consider complemented chief factors in $O_{p}(N)$. Since $O_{p}(N)=N \cap O_{p}(G)$, there are two cases to consider: $O_{p}(N)=$ $O_{p}(G)$, and $O_{p}(N)<O_{p}(G)$.

For the first case, put $P=O_{p}(G)$, and let $Q$ be the Frattini subgroup of $P$. Then $Q<\varphi N$, and again by Theorem 1 of Barnes (1972) it will be enough to consider complemented chief factors of $N$ between $P$ and $Q$. Since complemented chief factors of $G$ between $P$ and $Q$ correspond to maximal submodules of $P / Q$, regarded as a $Z_{p}[G]$-module, and since $G / C_{G}(P / Q)$ is a $p$-nilpotent group, we can conclude from Lemma 6.1 of Srinivasan (1960) that all the chief factors of $G$ between $P$ and $Q$ have $p^{\prime}$ absolute degree. The arguments of Hawkes (1970) then give that all the chief factors of $N$ between $P$ and $Q$ have $p^{\prime}$ absolute degree, finishing the proof in this case.

Now suppose $O_{p}(N)<O_{p}(G)$. Setting $P=O_{p}(G), R=O_{p}(N)$, we have $P / R$ cyclic of order $p$, and a central chief factor of $G$. Again, put $Q=\varphi P$, and also 
$S=\varphi R$. Then $P>R \geqslant Q \geqslant S$, and we want to show that the complemented chief factors of $N$ between $R$ and $S$ have $p^{\prime}$ absolute degree: again this is equivalent to all chief factors of $N$ between $R$ and $S$ having $p^{\prime}$ absolute degree. Since $G \in Y$, all the chief factors of $G$ between $Q$ and $R$ have $p^{\prime}$ absolute degree. If we set $H=P / S$, then $H^{\prime} \leqslant Q / S, H^{\prime}$ is elementary abelian, and as a $\mathbf{Z}_{p}[G]$-module it is a homomorphic image of $(P / Q) \otimes_{\mathbf{z}_{p}}(P / R)$. But $P / R$ is trivial as a $\mathrm{Z}_{p}[G]$-module, and so $H^{\prime}$ is a homomorphic image of $P / Q$. Since $p$ th powering is a $G$-homomorphism on $H / H^{\prime}$, we deduce that every chief factor of $G$ between $R$ and $S$ is isomorphic (as $\mathrm{Z}_{p}[G]$-module) to one between $P$ and $Q$. Again the arguments of Hawkes (1970) finish the proof.

We have now established that $Y$ is a Fitting class. To see that $Y$ is not a formation we observe that if it were, the groups of nilpotent length three in it would form a subgroup closed class (by Theorem 2 of Bryce and Cossey (1972)). Let $q$ be a prime such that $p$ divides $q-1$ (that such $q$ exist follows from Dirichlet's Theorem) and let $U$ be a faithful irreducible $\mathbf{Z}_{q}[C]$-module, where $C$ is a cyclic group of order $p$. Let $U^{*}$ be the contragredient of $U$ (see Curtis and Reiner (1962) Definition 43.7), and let $A$ be the group constructed from $U$ and $U^{*}$ as in Huppert (1967) Hilfssatz 6.7.22. Then $A$ is extraspecial, and the semidirect product $A C$ has a faithful irreducible module $M$ over $\mathbf{Z}_{p}$ whose absolute degree is $q$. But $\boldsymbol{M}_{U C}$ has an irreducible component of absolute degree $p$, and so $M A C \in \mathrm{Y}$ but $M U C \notin \mathrm{Y}$, giving the result.

We finish with some observations. Firstly, $\mathbf{X}$ could have been replaced by $\mathbf{S}_{p}, \mathbf{S}_{p} \mathbf{S}_{p}, \mathbf{S}_{p}$ : the proof becomes a bit more cumbersome, but is essentially the same. Secondly, we could use the Fitting formations of Berger and Cossey (1978) as our model to obtain a slightly different example.

The work for this paper was done during a brief but rewarding stay at Mainz University.

\section{References}

D. W. Barnes (1972), 'On complemented chief factors of finite soluble groups', Bull. Austral. Math. Soc. 7, 101-104.

T. R. Berger and John Cossey (1978), 'More Fitting formations', J. Algebra 51, 573-578.

R. A. Bryce and John Cossey (1972), 'Fitting formations of finite soluble groups', Math. Z. 127, 217-223.

R. A. Bryce and John Cossey (1974), 'Metanilpotent Fitting classes', J. Austral. Math. Soc. 17, 285-304.

C. W. Curtis and I. Reiner (1962), Representation theory of finite groups and associative algebras (Interscience, New York).

Trevor O. Hawkes (1970), 'On Fitting formations', Math. Z. 117, 177-182. 
B. Huppert (1967), Endliche Gruppen I (Springer, Berlin-Heidelberg-New York).

B. Srinivasan (1960), 'On the indecomposable representations of a certain class of groups', Proc. London Math. Soc. (3) 10, 497-513.

Department of Pure Mathematics

Faculty of Science

Australian National University

Canberra, A.C.T. 2600

Australia 